\title{
Estimation of Equivalent Thermal Conductivity for Electrical Windings with High Conductor Fill Factor
}

\author{
Sabrina Ayat \\ Electrical \& Electronic Systems Group \\ Safran Tech \\ Châteaufort, France \\ sabrina.ayat@safrangroup.com
}

\author{
Haipeng Liu \\ Electrical Energy Management Group \\ University of Bristol \\ Bristol, United Kingdom \\ liuhpbit@gmail.com
}

\author{
Mehmet Kulan and Rafal Wrobel \\ Centre for Advanced Electrical Drives \\ Newcastle University \\ Newcastle upon Tyne, United Kingdom \\ m.c.kulan@,newcastle.ac.uk \\ rafal.wrobel@newcastle.ac.uk
}

\begin{abstract}
In order to improve accuracy and reduce model setting up and solving time in the thermal analysis of electrical machines, the multi-material winding region is frequently homogenized. A typical electrical winding is an amalgam of the conductor material, electrical insulation, impregnation and imperfections like air cavities, which depend on the impregnation material and technique, among other factors. This makes the winding assembly one of the most challenging region in thermal design of electrical machines. This paper presents an analytical method for estimating the equivalent thermal conductivity of impregnated windings formed with round profile conductors. The existing analytical methods are limited to windings with conductor fill factor up to $40 \%$. However, the ongoing development in fabricating windings enabled winding arrangements with much higher conductor fill factors, up to $\mathbf{8 5 \%}$. Such high conductor fill is very desirable as it reduces the winding DC power loss while improving the thermal path for the generated heat. Thus, providing a reliable analytical approach for the estimation of the equivalent thermal conductivity, applicable across a wide range of conductor fill factors, is very desirable. This paper presents a detailed description of the proposed approach, supplemented with measured data from tests on hardware winding material samples (WSs). An illustration of a practical use of windings with high conductor fill factors is also provided, highlighting the benefits of such configuration.
\end{abstract}

Keywords-Winding region, homogenization, thermal conductivity, electrical machine, hardware testing.

\section{INTRODUCTION}

The stator-winding assembly is usually attributed with the dominant power loss component within the machine body [1]-[5]. A well-informed design of the winding region is therefore a pre-requisite of compact, high-power-density and high-efficiency machine solutions, such as these adopted in aerospace applications. Recent developments in the field of electrical machine design focus on solutions with improved thermal performance and reduced-loss generation, such as high conductor fill factor compressed coils [6]-[11].

The existing methods for estimating of the winding's thermal parameters must be adapted to the ongoing evolution and improvement in fabricating of the electrical windings [1][4]. The existing analytical methods for deriving of the equivalent thermal conductivity for windings formed with round profile conductors are limited to conductor fill factor $(F F)$ up to $40 \%$ [2]. Alternative techniques of deriving the winding equivalent thermal conductivity employ experimental or numerical methods. The analytical methods developed for the rectangular and Litz wire based winding topologies make use of lumped-parameter thermal equivalent circuit (TEC) approach to estimate the winding equivalent thermal conductivity [3], [4]. These methods account for thermal path of the heat flux across the impregnated conductors. Such approach ensures improved accuracy as compared with the existing analytical approaches, which are predominantly based on volumetric contribution of the constituent materials within the winding amalgam [2]. a)

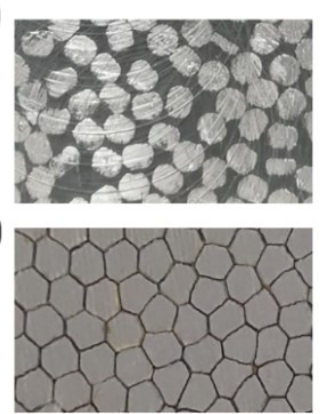

c)

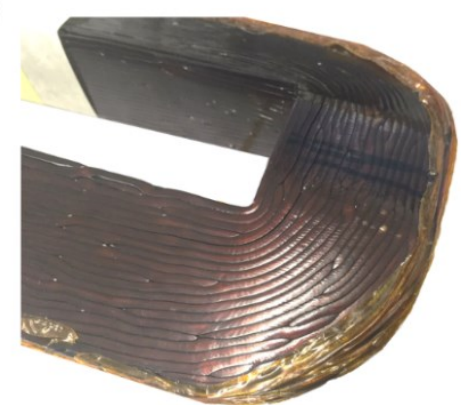

Fig. 1 Impregnated winding samples, a) Close-up view on a conventional winding $(F F=55 \%)[2]$, b) Close-up view on a compressed winding $(F F=80 \%)$ [7], c) A pre-compressed coil exemplar [7]

This work is focused on developing an analytical approach for the estimation of the equivalent thermal conductivity of impregnated electrical windings formed with round conductors. One of the key challenges associated with the development of a method for estimating the equivalent thermal conductivity for windings with high conductor fill factor using TEC, is to understand how the individual conductors deform when compressed in a coil forming fixture [6]-[11]. The high conductor fill factor leads to plastic deformation of the conductors' profile from round to polygonal as shown in Figs. 1a) and 1b). Also, Fig. 1c) shows an example of a complete preformed coil with a high conductor fill factor $(80 \%)$ for a high specific output machine design [7]. The winding technique employed here enables geometrically accurate coil fabrication. It is expected that the manner, in which the individual conductors deform depends on the overall coil geometry and the winding compression technique.

In this investigation, a simplified mechanical analysis has been carried out to gain an insight into the mechanical aspects of the winding construction with high conductor fill factor. The approach used here employs a two-dimensional (2D) mechanical finite element (FE) analysis similar to that presented in [3], [4]. The theoretical findings from the FE analysis have been used to inform the proposed technique for estimating the equivalent thermal conductivity using the TEC approach. The method relies on an averaged conductor profile. The theoretical predictions from the new method have been compared with data from the existing numerical 
technique showing good correlation. Also, the theoretical body of work has been supplemented with experimentally derived results from tests on several winding samples. Finally, a case study thermal analysis of a machine for aerospace application is presented, highlighting the importance of reliable thermal data for the analysis of high specific output electrical machines. The benefits of using winding formed with compressed conductors are also emphasized.

\section{WINDING MATERIAL SAMPLES}

Several winding samples (WSs) have been manufactured for the purpose of this research. Figs. 1 and 2 present the selected winding samples together with relationship between them. The winding samples were carefully chosen to differ in terms of conductor fill factor and impregnation material. The variety of the analyzed samples enables here a more generic insight into the composite winding thermal properties and their dependence on the geometrical, material and manufacturing factors. Table I presents basic data for the analyzed winding samples.

A heat flux meter approach has been employed in this investigation to measure the thermal conductivity of the WSs [1]-[5]. During the test, a known heat transfer rate is applied by dissipating a pre-set power in a power resistor. The tested winding sample is placed on a temperature-controlled cold plate inside a thermally insulated chamber to ensure a unidirectional heat transfer. Fig. 3 presents a photograph of the experimental set-up, before surrounding the winding sample with thermal insulating material.

TABLE I. BASIC DATA FOR THE ANALYZED IMPREGNATED WINDING SAMPLES

\begin{tabular}{lllllll}
\hline Winding sample (WS) & I & II & III & IV & V & VI \\
Fill factor $F F[\%]$ & 55 & 60 & 66 & 60 & 55 & 80 \\
Conductor radius [mm] & 0.8 & 0.35 & 0.8 & 0.8 & 0.8 & 0.75 \\
Conductor material & $\mathrm{Cu}$ & $\mathrm{Cu}$ & $\mathrm{Al}$ & $\mathrm{Cu}$ & $\mathrm{Al}$ & $\mathrm{Al}$ \\
Electrical insulation & 1 & 1 & 1 & 1 & 2 & 1 \\
material & & & & & & \\
Impregnation material & 3 & 4 & 4 & 4 & 4 & - \\
Electrical insulation & 35 & 35 & 35 & 35 & 3.5 & 42
\end{tabular}

thickness $[\mu \mathrm{m}]$

${ }^{1}$ Polyamide-imide enamel, ${ }^{2}$ Aluminium oxide, ${ }^{3}$ Varnish (Ultimeg

2000/380), ${ }^{4}$ Epoxy resin (Epoxylite EIP 4260).

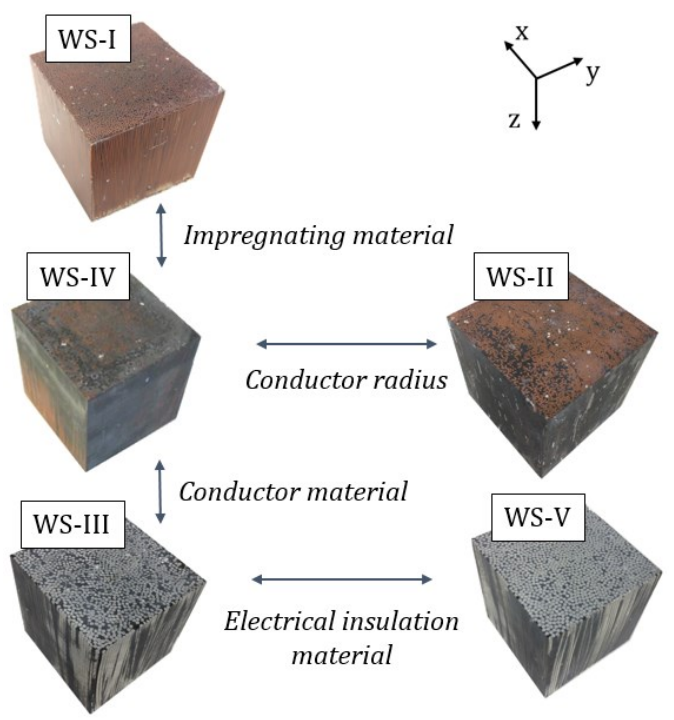

Fig. 2 Relationship between the analyzed impregnated WSs

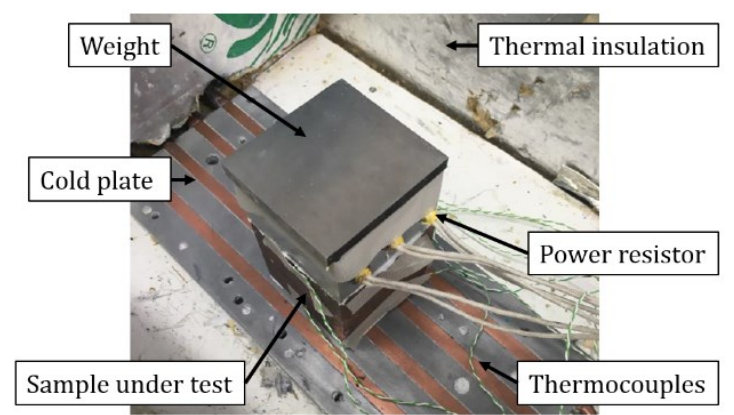

Fig. 3 Experimental set-up before surrounding the winding sample with thermal insulating material

A set of thermocouples are embedded in the sample on each face to allow the average temperature measurements to be taken. A thermally-conductive paste is applied to both interfaces to improve the contact heat transfer. A detailed description of the experimental set-up together with the testing procedure, is provided in the authors' previous work [1]-[4]. The thermal conductivity is derived from,

$$
k=\frac{\left(R_{\Omega} I^{2}\right) l}{\left(T_{1}-T_{2}\right) A}=\frac{P_{\Omega} \cdot l}{\left(T_{1}-T_{2}\right) A}
$$

where $I$ represents the DC current excitation applied to the resistor $R_{\Omega}, l$ the distance between measuring points for temperature $T_{1}$ and $T_{2}$, and $A$ the cross-section area of the sample through which the heat is transferred. The applied current and voltage are both measured when the steady-state is reached in order to account for the effect of temperature on the ohmic resistance value. Table II includes a set of measured thermal conductivities for each of the selected winding samples, with measurement uncertainty estimated using the propagation of error formula [16].

TABLE II. MEASURED SET OF THERMAL CONDUCTIVITIES FROM TESTS ON WINDING SAMPLES

\begin{tabular}{cccc} 
Winding sample & $\begin{array}{c}k_{x} \\
{[\mathrm{~W} /(\mathrm{m} \cdot \mathrm{K})]}\end{array}$ & $\begin{array}{c}k_{y} \\
{[\mathrm{~W} /(\mathrm{m} \cdot \mathrm{K})]}\end{array}$ & $\left.\begin{array}{c}k_{z} \\
{[\mathrm{~W} /(\mathrm{m} \cdot \mathrm{K})}\end{array}\right]$ \\
I & $2.1 \pm 0.1$ & $2.1 \pm 0.1$ & - \\
II & $2.0 \pm 0.1$ & $2.5 \pm 0.1$ & $166 \pm 6$ \\
III & $2.4 \pm 0.1$ & $2.3 \pm 0.1$ & $151 \pm 5$ \\
IV & $2.5 \pm 0.1$ & $2.7 \pm 0.1$ & $215 \pm 8$ \\
V & $6.5 \pm 0.2$ & $6.3 \pm 0.2$ & $167 \pm 6$ \\
VI & $2.0 \pm 0.1$ & $2.0 \pm 0.1$ & - \\
\hline
\end{tabular}

As expected, the thermal conductivity in $\mathrm{x}$-axis is similar to the one in y-axis for round conductors. The use of aluminum conductor instead of copper has limited impact on the equivalent winding thermal conductivity, WSs II and III. The use of epoxy resin impregnation has shown improved overall thermal properties of the impregnated winding samples, e.g. WSs I and IV. This results from better material thermal properties and improved encapsulating properties for the epoxy impregnation as compared with the varnish impregnation. The limiting factors are the impregnation and electrical insulation materials. This is confirmed by the difference between WSs III and V. These two samples use identical conductors with different type of electrical insulation material (conductor coating). Here, the use of aluminum oxide instead of enamel results in a $170 \%$ increase in the winding thermal conductivity. The compressed winding sample, WS VI, offers an equivalent thermal conductivity comparable to WSs I, II, III and IV without any form of impregnation. This is of interest when optimizing the machine manufacturing process. Indeed, replacing the 
winding impregnation by the winding compression could decrease the machine construction time and resources, i.e. time and equipment required to cure the impregnation, and cost, i.e. material and processing cost.

\section{CONVENTIONAL WINDINGS FORMED WITH ROUND-PROFILE CONDUCTORS}

The numerical and analytical methods have been commonly used to derive the equivalent winding thermal conductivity of impregnated winding formed with roundprofile conductors. The numerical method makes use of FE analyses, while the proposed method makes use of the TEC approach.

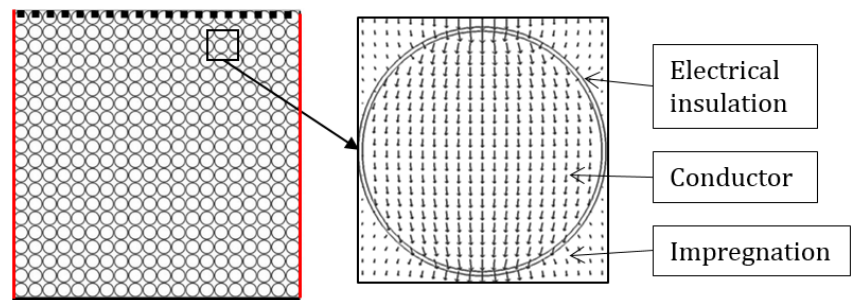

y $\quad$..... Fixed heat flux boundary condition

$\overrightarrow{\mathrm{x}}=$ Fixed temperature boundary condition

- Adiabatic boundary condition

Fig. 4 2D thermal FE model representation of a winding sample together with boundary conditions; a close-up view of an individual conductor with electrical insulation and impregnation is also shown with the heat flux.

\section{A. Numerical Method}

Fig. 4 presents a 2D FE representation of a winding sample cross-section. Here, the aligned conductor lay is adopted for the FE model [2]. A fixed temperature boundary condition is used to represent the cold plate, while a fixed heat flux boundary condition is used to embody the known heat flow from the power resistor. The remaining surfaces of the impregnated winding sample are defined with adiabatic boundary conditions, emulating the adopted testing procedure.

A close-up view of an individual conductor with electrical insulation and impregnation is also shown in Fig. 4, with the sub-regions highlighted. The model representation used in the FE analysis may be reduced to one individual conductor. An initial analysis with the complete winding sample and reduced-order one-conductor models has shown insignificant discrepancy between the results, due to the structure periodicity, for all conductor types [3].

\section{B. Existing analytical method}

The existing analytical method is based on the analytical expression for estimating the equivalent thermal conductivity $k_{w}$ of winding formed with round conductors presented in detail in [2]. Here, a simplified form of the analytical expression is provided only,

$$
k_{w}=k_{a} \frac{\left(1+v_{c}\right) k_{c}+\left(1-v_{c}\right) k_{a}}{\left(1-v_{c}\right) k_{c}+\left(1+v_{c}\right) k_{a}}
$$

Where $k$ is the thermal conductivity, $v$ is the material volume ratio, and the subscripts $c$ and $a$ refer to the conductor and electrical insulation/impregnation amalgam, respectively. This commonly-used analytical method has been investigated in [2], showing its limited applicability for windings with the conductor packing factor up to $40 \%$. Therefore, this method

is used in this work to highlight the merits of the proposed analytical approach.

\section{Proposed analytical method}

The proposed method makes use of the TEC theory to derive the equivalent thermal conductivity of winding formed with round conductors. Fig. 5a) shows a simplified model representation of a half of conductor cross-section together with electrical insulation and impregnation. The derivation of the equivalent thermal resistance for the impregnation region represented in Fig. 5a) is not trivial, due to the difficulty of setting-up adequate boundary conditions for such geometry. The proposed method makes use of an equivalent thermal resistance for the equivalent half hollow cylinder with identical cross-section area $A_{i}$, Fig. 5b). Fig. 5c) presents the TEC associated with the cylindrical single conductor representation, together with the nomenclature used.

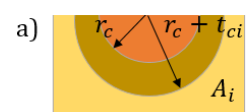

b)

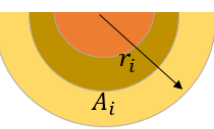

c)

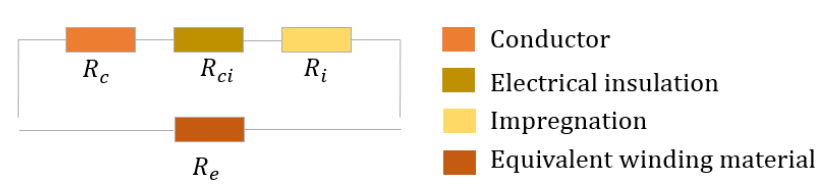

Fig. 5 Schematic model representation of a half conductor/electric insulation/impregnation amalgam, a) initial model representation, b) surrogate model representation, c) lumped-parameter TEC, and nomenclature used.

Here, $R_{c}, R_{c i}, R_{i}$ and $R_{e}$ correspond to the thermal resistance of the conductor, electrical insulation, impregnation and equivalent homogenized materials, respectively. From Figs. 5a) and 5b), $A_{i}$ can be expressed as follows:

$$
A_{i}=\frac{\pi r_{i}^{2}}{2}
$$

where $r_{i}$ is the radius of the impregnation hollow cylinder in the cylindrical winding model representation. The fill factor is defined as:

$$
F F=\frac{\pi r_{c}^{2}}{2 A_{i}}
$$

where $r_{c}$ conductor radius. Hence, $r_{i}$ can be expressed using the following expression:

$$
r_{i}=\frac{r_{c}}{\sqrt{F F}}
$$

The TEC model and associated choices of equivalent thermal resistances are based on observations from the heat flux behavior on FE models. According to observations made from Fig. 3, the heat fluxes across the electrical insulation and impregnation have a radial direction, while the heat flux across the conductor flows along the y-axis. Therefore, $R_{c i}$ and $R_{i}$ are defined as radial thermal resistances, while $R_{c}$ requires the derivation of a unique expression based on the conductor geometry. Details of the mathematical derivation of $R_{c}, R_{c i}, R_{i}$ and $R_{e}$ are provided in Appendix, including derivation of $k_{w}$. A closed-form equation is provided as follows: 


$$
k_{w}=\frac{1}{\frac{\ln \frac{r_{C}}{\left(r_{c}+t_{c i}\right) \sqrt{F F}}}{k_{i}}+\frac{\ln \frac{r_{c}+t_{c i}}{r_{c}}}{k_{c i}}+\frac{1}{k_{C}}}
$$

The assumptions made in the derivation of $k_{w}$, such as the radial heat transfer across the electrical insulation material, is only valid for $k_{c}>>k_{c i}$. This is generally the case for the winding amalgams. However, if uncommon materials were to be used, the formula might require a revision.

\section{Initial Comparison}

In order to demonstrate applicability and limitations of proposed method, $k_{w}$ has been estimated for WS-I's configuration. Fig. 6 presents variation of the equivalent thermal conductivity versus conductor fill factor assuming fixed cross-section of an individual conductor. Each plot corresponds to a specific value of $k_{i}$ from $0.5 \mathrm{~W} /\left(\mathrm{m} .{ }^{\circ} \mathrm{C}\right)$ to $1.1 \mathrm{~W} /\left(\mathrm{m} .{ }^{\circ} \mathrm{C}\right)$. The thermal conductivity values of conductor material and electrical insulation are kept constant, $k_{c}=380$ $\mathrm{W} /\left(\mathrm{m} .{ }^{\circ} \mathrm{C}\right)(\mathrm{Cu}$ conductors $)$ or $k_{c}=215 \mathrm{~W} /\left(\mathrm{m} .{ }^{\circ} \mathrm{C}\right)(A l$ conductors), and $k_{c i}=0.27 \mathrm{~W} /\left(\mathrm{m} .{ }^{\circ} \mathrm{C}\right.$ ) (Polyamide-imide enamel) or $k_{c i}=2.0 \mathrm{~W} /\left(\mathrm{m} .{ }^{\circ} \mathrm{C}\right.$ ) (Aluminum oxide), respectively.

The proposed analytical method shows very good correlation with the numerical method, while the limited applicability of the existing analytical method at high conductor fill factor is confirmed, in particular for conductor fill factors superior to $55 \%$. The existing analytical method has been originally developed for two-material composites in [15], and has been adapted in [2] to three-material amalgams. Therefore if $k_{i}$ is dissimilar to $k_{c i}$, the initial assumptions made in [15] considering a two-material composite may no longer be valid.

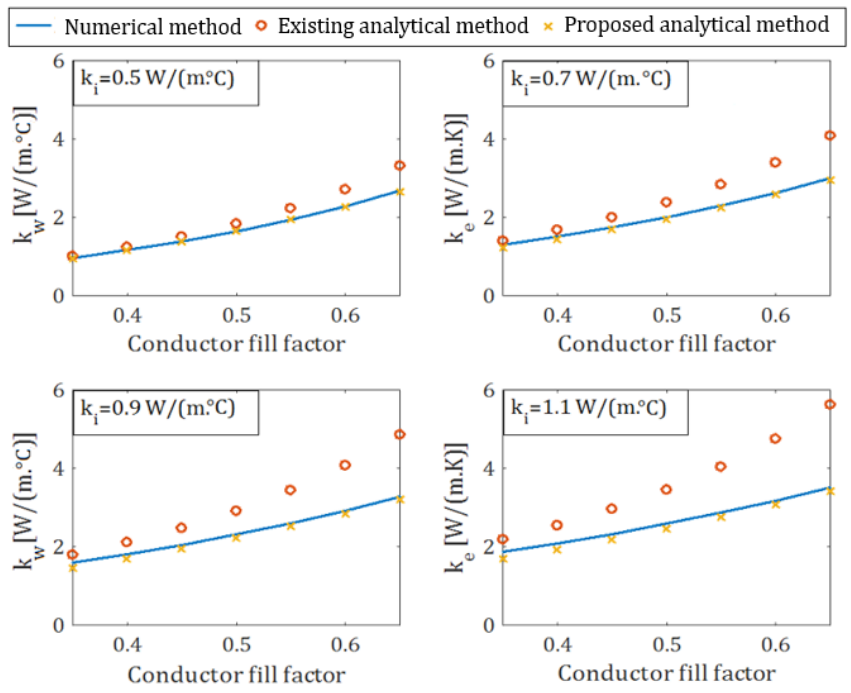

Fig. 6 Predicted thermal conductivity vs. conductor fill factor for different impregnation 'goodness'

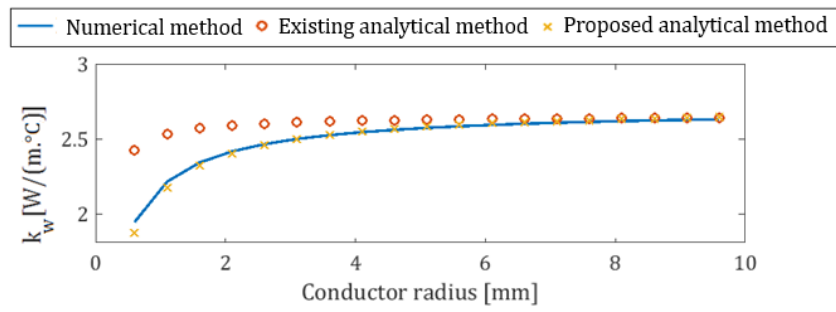

Fig. 7 Thermal conductivity vs. conductor radius
Fig. 7 presents the variations of the equivalent thermal conductivity versus conductor radius assuming a fixed fill factor of the winding assembly, $F F=55 \%$. On the one hand, the proposed analytical method shows again very good correlation with the results from the existing analytical method. On the other hand, the applicability of the existing analytical method appears limited when the electrical insulation thickness $t_{c i}$ is not negligible compared to the conductor radius $r_{c}$. In this case, the winding assembly cannot be considered a two-material composite, and the initial assumptions made in [15] may again no longer be valid.

Table III presents the measured and calculated thermal conductivity values from WSs II to V. The measured thermal conductivity values in the $\mathrm{x}$ - and $\mathrm{y}$ - axes have been averaged to obtain a single value for the comparison. The impregnation thermal conductivity values $k_{i}$ used in the FE model have been tuned to match the measured results. Then, these calibrated $k_{i}$ have been used to calculate the winding equivalent thermal conductivity $k_{w}$ using the proposed analytical method. The results correlate well, with a maximum deviation of $10 \%$ observed between WS II's calculated data.

TABLE III. AVERAGE MEASURED AND CALCULATED SETS OF THERMAL CONDUCTIVITIES OF WINDING SAMPLES

\begin{tabular}{lllll}
\hline Winding sample & II & III & IV & V \\
$k_{w, \text { measured (average) }}\left[\mathrm{W} /\left(\mathrm{m} .{ }^{\circ} \mathrm{C}\right)\right]$ & 2.25 & 2.35 & 2.6 & 6.4 \\
$k_{w, \text { calculated-FE method }}\left[\mathrm{W} /\left(\mathrm{m} .{ }^{\circ} \mathrm{C}\right)\right]$ & 2.2 & 2.35 & 2.6 & 6.4 \\
$k_{w, \text { calculated-proposed method }}^{1}\left[\mathrm{~W} /\left(\mathrm{m} .{ }^{\circ} \mathrm{C}\right)\right]$ & 2.0 & 2.35 & 2.55 & 6.3
\end{tabular}

${ }^{1}$ Calculated using the impregnation thermal conductivity derived from $\mathrm{FE}$ calibration

\section{WINDINGS FORMED WITH ROUND-PROFILE COMPRESSED CONDUCTORS}

\section{A. Mechanical Analysis}

A structural FE approach has been used here to derive deformed winding samples for different conductor fill factors. A plastic deformation of compressed windings can be modelled using an appropriate FE solver, e.g. a quasi-static explicit dynamic. Such analysis is effectively based on a dynamic phenomenon as is in the physical structure. The compression of a coils/winding sample is highly non-linear computational problem due to the geometrical, material stress-strain relationship and frictional body interactions between the individual conductors. The quasi-static explicit dynamic analysis is preferred here in contrast to a more conventional static structural FE approach.

A FE model of a 15-turn coil with aluminium conductors has been solved to mimic the winding sample compression, Fig. 8. A velocity boundary conditions have been applied on the steel punches positioned on the sides of the winding segment to compress the conductors. The approach employed here is similar to that presented in [10], used to derive deformed conductor geometry for different slot fill factors.

Since FE boundary conditions and solver settings are of significant importance for the accuracy of the simulations, conservation of energy in the overall system needs to be monitored to ensure that the mesh deformation is realistic [10]. 


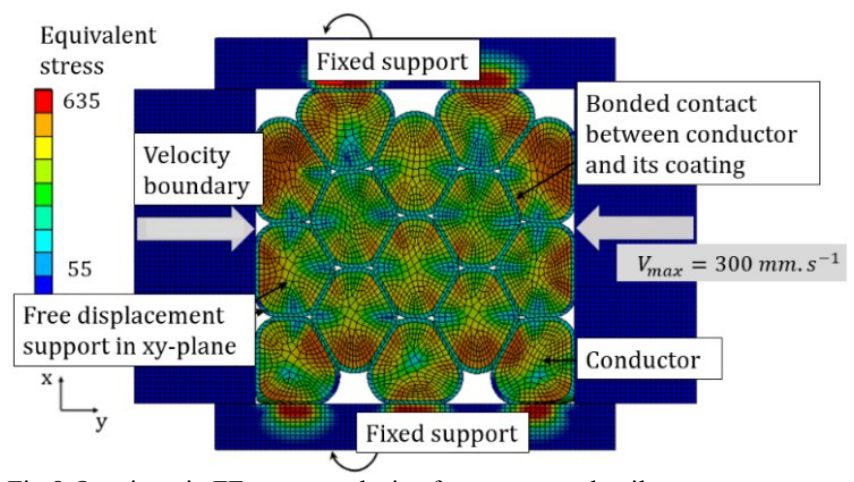

Fig.8 Quasi-static FE stress analysis of a compressed coil

This has been achieved in Fig. 8 with a maximum fill factor of $80 \%$. Theoretical predictions suggest that further compression of the geometry would result in the insulation failure assuming a polymer-based wire insulation's stressstrain curve. The realistic compressed winding geometries obtained from the mechanical analysis have been used for the estimation of the equivalent thermal conductivity of the compressed windings at various fill factors.

\section{B. Thermal analysis}

Four initial examples of compacted windings with different conductor fill factors have been selected from the mechanical $\mathrm{FE}$ analysis to investigate their equivalent thermal properties, Fig. 9. The FE thermal model definition employed here is similar to that discussed earlier in Section III. However, for the compressed windings, the conductor fill factor is too high to reduce the FE thermal model to just one conductor, Fig. 4. Consequently, a model definition encompassing twoconductors has been used as shown in Fig. 9. Here, the first, complete conductor is placed in the center while four quarters of the second conductor are located in each corner of the FE model. It has been assumed in the FEAs that thermal conductivity of air is equal to $k_{\text {air }}=0.036 \mathrm{~W} /\left(\mathrm{m} .{ }^{\circ} \mathrm{C}\right)$. As previously mentioned, the conductors may deform in an irregular manner, depending on the adopted compression approach. As a consequence, the equivalent winding thermal conductivity may become anisotropic. Fig. 10 presents the 2D FE representation of the compressed winding cross-section, highlighting the temperature distribution within the conductors.

Fig. 11 presents the calculated thermal conductivity, for both $\mathrm{x}$ - and y-axes. The measured data point from tests on the compressed winding sample is also shown. The results confirm the expected thermal anisotropy, which depends on the way the conductors deform. a)

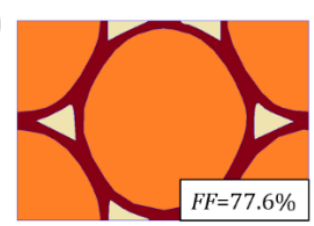

c)

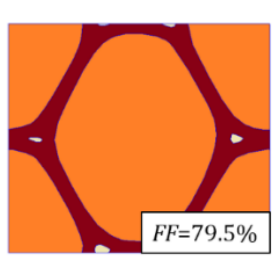

Conductor

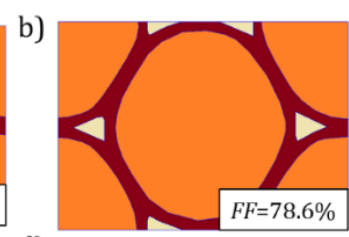

d)

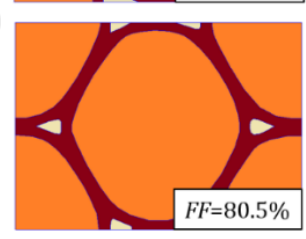

Electrical insulation Air
Fig.9 Compressed winding geometries selected for the thermal analysis

a)

b)
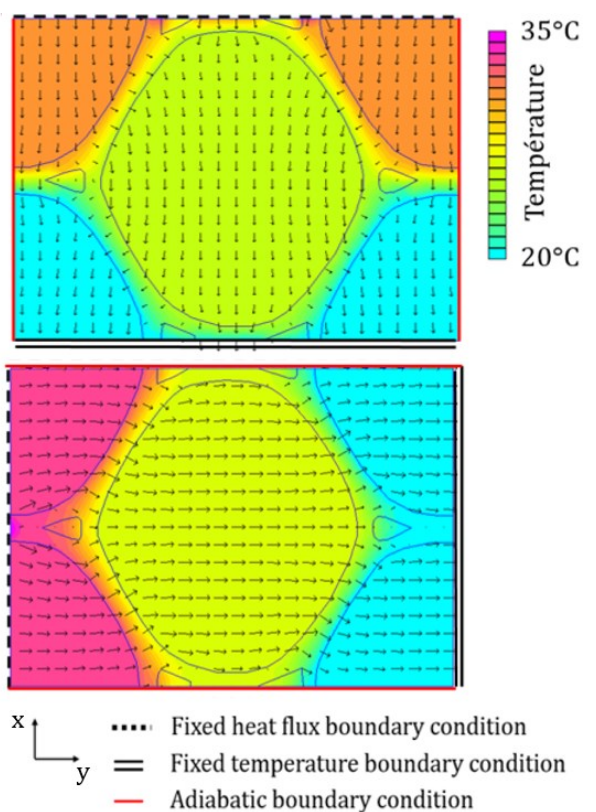

Fig.10 2D thermal FEA model representation of a winding sample together with boundary conditions, a) $\mathrm{x}$-axis, b) $\mathrm{y}$-axis.

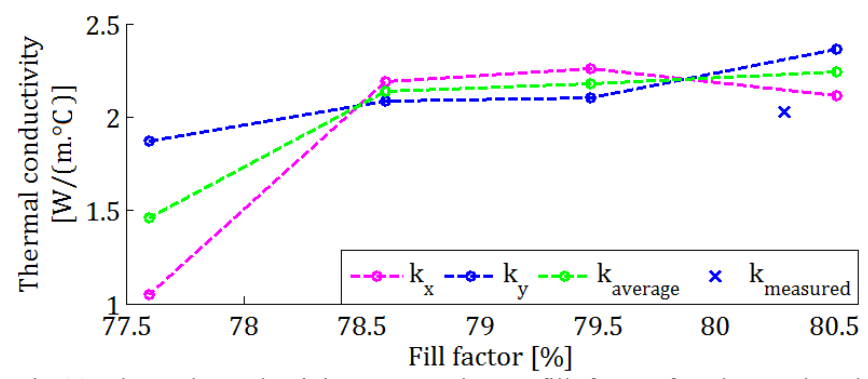

Fig.11 Thermal conductivity vs. conductor fill factor for the analyzed winding examples

The averaged winding thermal conductivity increases with the winding fill factor. The measured value appears lower than the theoretical estimates. This is due to the perfect contact between the electrical insulation conductors assumed in the FE model. Indeed, after the mechanical analysis, the electrical insulation regions that are in contact merge together forming a 'merged electrical insulation region'. In practice, the interface between the regions is imperfect and impacts the winding thermal behavior.

An equivalent thermal conductivity could be defined for the 'merged electrical insulation region', accounting for the thermal contact resistance. In the analyzed case, the equivalent thermal conductivity is equal to $k_{e}=0.2 \mathrm{~W} /\left(\mathrm{m} .{ }^{\circ} \mathrm{C}\right)$ This is to calibrate the FE model with the measured data. Due to the change in conductor shape during compression, and the direct contact between conductors, the formula developed for conventional windings formed with round conductors cannot be directly applied to compressed windings. An analysis based on the observation of the heat flux within the winding, as presented in Section III, could be employed in developing of the analytical method for the compressed windings. In particular, the air gaps between conductors observed on Fig. 8 tends to have triangular shapes, and could therefore be easily represented via a TEC approach. More work is nonetheless required to account for the conductor deformation. 


\section{ILLUSTRATION}

A radial-flux interior permanent magnet (IPM) machine designed for a steering application has been investigated to illustrate the effect of using compressed winding on the machine power output capability. Fig. 12 presents the machine structure with all the active regions indicated. The machine's stator has an open-slot topology, which permits the insertion of the compressed windings. To improve the heat extraction from the winding body to the machine's periphery, ensure an adequate electrical insulation and a good mechanical rigidity, the complete stator-winding assembly is impregnated with varnish and housed in a standard flange mounted aluminum frame. Basic machine data is listed in Table IV.

\begin{tabular}{ll} 
TABLE IV. BASIC MACHINE DATA & \\
\hline Outer Diameter/ Active Length & $180 \mathrm{~mm} / 160 \mathrm{~mm}$ \\
Number of poles/slots & $10 / 12$ \\
Base rotational speed & $4600 \mathrm{rpm}$ \\
Rated torque & $62 \mathrm{~N} \cdot \mathrm{m}$ \\
DC link voltage & $340 \mathrm{~V}$ \\
Cooling type & Water jacket \\
Cooling temperature & $70^{\circ} \mathrm{C}$ \\
Electrical insulation class & $\mathrm{H}\left(180^{\circ} \mathrm{C}\right)$ \\
\hline
\end{tabular}

A three-dimensional (3D) TEC has been built to predict the machine steady state and transient torque output capability, using cuboidal and arc-segment elements [1], [17]. The active and end winding assemblies are modelled based on the homogenized winding thermal properties, predicted using (8). The thermal properties of other materials, including laminated stator and rotor core packs (M270-35A), PM array ( $\mathrm{SmCo})$, shaft (Stainless Steel), end-cap and housing frame (Aluminium) are obtained from the available literature [18], [19]. More details about the thermal network are available in the author's previous work [6], [8].

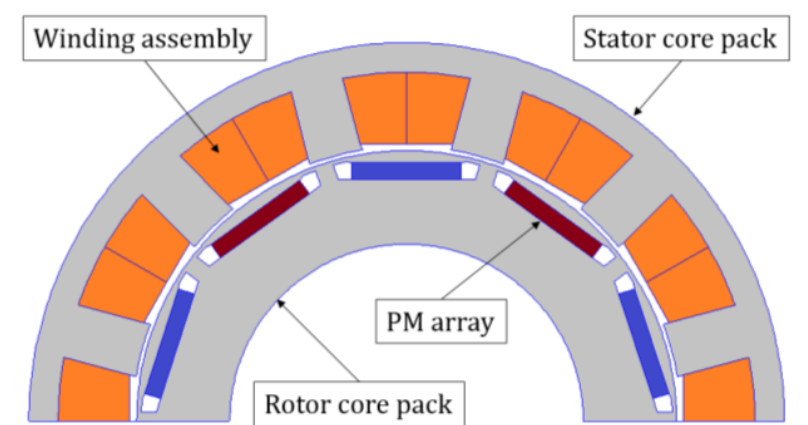

Fig. 12 A schematic drawing of the investigated PM machine assembly with active regions indicated.

TABLE V. CALCULATED DATA FOR THE SELECTED EXAMPLES

\begin{tabular}{llll}
\multicolumn{4}{c}{ TABLE V. CALCULATED DATA FOR THE SELECTED EXAMPLES } \\
\hline$F F[\%]$ & $r_{c}[\mathrm{~mm}]$ & $k_{w}\left[\mathrm{~W} /\left(\mathrm{m} .{ }^{\circ} \mathrm{C}\right)\right]$ & $c_{S, w}\left[\mathrm{~J} /{ }^{\circ} \mathrm{C}\right]$ \\
65 & 0.74 & 2.5 & 483 \\
75 & 0.79 & 3.3 & 442 \\
85 & 0.85 & 4.6 & 408 \\
\hline
\end{tabular}

The impact of three different conductor fill factors $(65 \%$, $75 \%$ and $85 \%$ ) on the machine continuous and transient thermal envelopes has been investigated. Only the conductors' radius $r_{c}$ is varied, i.e. the number of turns and winding connections are kept unchanged. The equivalent winding thermal conductivity values have been calculated for the three examples, and are listed in Table V. The winding specific heat capacity $c_{s, w}$ has been calculated using (8), [2],

$$
c_{s, w}=\frac{P F\left(\rho_{c} c_{s, c}-\rho_{i} c_{s, i}\right)+\rho_{i} c_{s, i}}{P F\left(\rho_{c}-\rho_{i}\right)+\rho_{i}}
$$

where $P F$ is the packing factor, i.e. the ratio of wire to complete winding, $\rho$ and $c_{s}$ are the density and the specific heat capacity, respectively. According to [2], the electrical insulation can be neglected when calculating the winding equivalent specific heat capacity.

The ratio of equivalent $\mathrm{AC}$ and $\mathrm{DC}$ winding resistances, $\left(R_{a c} / R_{d c}\right)$ is expressed as follows:

$$
\left(\frac{R_{a c}}{R_{d c}}\right)_{T_{0}}(f)=\left(\left(\frac{R_{a c}}{R_{d c}}\right)_{T_{0,}, f_{\max }}-1\right)\left(\frac{f}{f_{\max }}\right)^{2}+1
$$

where $\left(\frac{R_{a c}}{R_{d c}}\right)_{T_{0}, f_{\max }}$ is the ratio of equivalent $\mathrm{AC}$ to $\mathrm{DC}$ resistances at a reference temperature $T_{0}$ and at a maximum excitation frequency $f_{\text {max }}$. The open-slot topology, required for the compressed winding assembly, is expected to results in elevated $\mathrm{AC}$ effects due to the rotor rotation for the conductors at the top of the slot. However, aluminum conductors are used in this machine. The high resistivity of aluminum will result in different $\mathrm{AC}$ and $\mathrm{DC}$ winding loss distributions than when copper conductors are used, with lower $\mathrm{AC}$ and higher DC winding loss components. The ratio has been kept constant for the selected examples, here, $\left(\frac{R_{a c}}{R_{d c}}\right)_{T_{0}, f_{\text {max }}}=3$. The winding AC power loss are still likely to be higher for windings with high fill factor if no particular measures are taken to reduce them. Future work will include analysis of the impact of the conductor radius on the $\mathrm{AC}$ winding power loss. The functional representation of the AC winding power loss temperature dependence, proposed in [20], has been used when updating the losses with temperature:

$$
P_{a c \mid T_{0}}=P_{d c \mid T_{0}}\left(1+\alpha\left(T-T_{0}\right)\right)+P_{d c \mid T_{0}} \frac{\left(\frac{R a c}{R_{d c}}\right)_{\mid T_{0}}-1}{\left(1+\alpha\left(T-T_{0}\right)\right)^{\beta}}
$$

where $\beta$ is the temperature coefficient for the ac loss component and $T_{0}$ is the temperature at which the DC power loss $P_{d c}$ has been calculated. The parameter $\beta$ has been derived from a curve fit of (10) to the winding AC power loss data derived from FEAs at two reference temperatures. The average winding temperature has been limited to $165^{\circ} \mathrm{C}$ in the continuous and transient thermal envelopes calculations.

Fig. 13 presents the calculated continuous and transient thermal envelopes for the machines with alternative conductor fill factors. The change in conductor fill factor has more impact on the machine transient torque capability than on the machine continuous torque capability, with an increase of approximately $15 \%$ observed on the complete machine torque/speed envelope when replacing a $65 \%$ fill factor with an $85 \%$, compared to an increase of approximately $21 \%$ at machine rotation speed inferior to $5000 \mathrm{rpm}$. At high rotational speed, the transient torque is constrained by the maximum DC link voltage, and not by the winding maximum temperature. Therefore, there are no difference in the transient torques for the investigated examples in this context. 


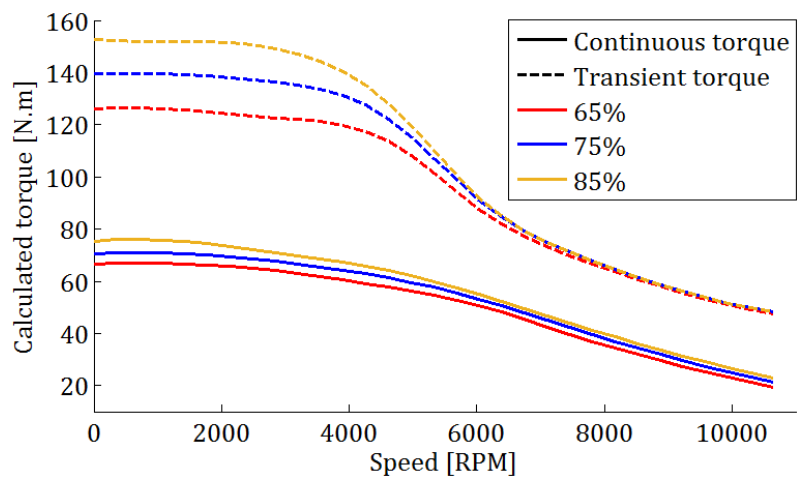

Fig. 13 Continuous and transient torque for machine alternatives with different conductor fill factors.

The torque speed envelope with winding thermal limit is influenced by the winding equivalent thermal conductivity, winding equivalent heat capacity and winding electrical resistance, all of which are simultaneously influenced by the conductor fill factor. To illustrate the influence of conductor fill factor on the winding thermal conductivity, and provide more details on the winding temperature distribution, steadystate FE thermal analyses have been completed on a single stator segment, Fig. 14. The winding-to-stator contact thermal contact resistance and the core thermal conductivity are kept unchanged for the three investigated examples. An equivalent convection boundary condition of $70{ }^{\circ} \mathrm{C}$ and 1000 $\mathrm{W} /\left(\mathrm{m}^{2} . \mathrm{K}\right)$ is applied to the stator outer boundary to account for the stator-to-water jacket thermal contact resistance and the water jacket cooling. A DC winding power loss of $70 \mathrm{~W}$ is input to the winding region for the three examples. The change in conductor fill factor has limited influence on the winding average temperature. However, there is a clear difference in the temperature gradient for windings with different conductor fill factors, Fig. 14. There is $11 \%$ reduction in hot spot temperature rise for winding with $85 \%$ conductor fill factor compared with that with $65 \%$ conductor fill factor. In practice, the winding loss will not be homogeneously distributed within the winding assembly, and therefore the difference in hot spot temperature is expected to be more important. The inhomogeneous winding loss distribution can be accounted for in a simple manner by subdividing the winding area into smaller sub-regions, with appropriate winding loss share [20].

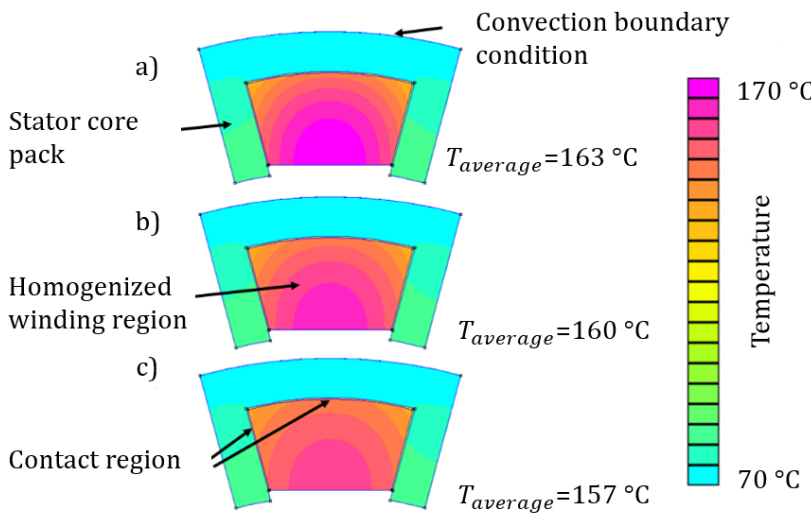

Fig. 13 Winding temperature distribution for stator segment with different conductor fill factors when same winding power loss are assumed. a) $F F=65 \%$. b) $F F=75 \%$. c) $F F=85 \%$

\section{VI.CONCLUSIONS}

This paper presents an analytical approach for the estimation of the equivalent thermal conductivity of impregnated electrical windings formed with round conductors. The formula has shown improved accuracy compared to the existing analytical techniques for conductor fill factors up to $75 \%$. The theoretical analysis has been supplemented with measured data from tests on hardware winding material samples. In this context, the validity of the calibrated thermal conductivity of impregnation for alternative winding configurations has been confirmed.

An insight into the mechanical and thermal aspects of winding constructions with high conductor fill factor has been gained. The use of a higher conductor fill factor results in a notable increased winding equivalent thermal conductivity and reduced winding DC power losses. The thermal conductivity value highly depends on the way the conductors deform during compression, and may be anisotropic depending on the adopted compression approach. First observations on the derived numerical data constitutes a first step into the development of a more generic analytical formula for compressed windings. In particular, the triangular shapes of the air gap between conductors after winding compression seem well-suited for a lumped-parameter TEC representation.

The use of the compressed conductor has been illustrated on a PM machine designed for a steering application. A notable increase in the machine continuous and transient torque output capability has been observed. This work used the improved conductor fill factor permitted by the compression to increase the conductor radius. Different winding modifications could also be considered, such as a change in number of turns. The investigated machine's duty cycle is a combination of continuous and transient operation. The analysis of the machine performance for a representative operating cycle would offer complete information concerning the total gain offered by compressed coil. For machines with short transient application, such as the auxiliary motors used to quickly restart the engine in Safran "more electric" twinengine helicopters [21], compressed windings can be considered as a promising arrangement.

\section{APPENDIX}

According to observations made from Fig. 4, the heat fluxes across the electrical insulation and impregnation have a radial direction, while the heat flux across the conductor flows along y-axis. The radial thermal resistance in a hollow cylinder, $R_{r, \text { hollow }}$, and half hollow cylinder, $R_{r, \text { hollow, half }}$ are obtained from the well-known solution of the heat conduction equation with zero heat generation and expressed as follows, respectively [14]:

$$
\begin{aligned}
& R_{r, \text { hollow }}=\frac{\ln \frac{r_{2}}{r_{1}}}{2 \pi k L} \\
& R_{r, \text { hollow }, \text { half }}=\frac{\ln \frac{r_{2}}{r_{1}}}{\pi k L}
\end{aligned}
$$

where $L, r_{1}$ and $r_{2}$ are the hollow cylinder height, inner and outer radius. The thermal resistances $R_{c i}$ and $R_{i}$, are derived from (13) and (14), 


$$
\begin{aligned}
& R_{i}=\frac{\ln \frac{r_{i}}{r_{c i}}}{\pi k_{i} L} \\
& R_{c i}=\frac{\ln \frac{r_{c i}}{r_{c}}}{\pi k_{c i} L}
\end{aligned}
$$

The thermal resistance across a half cylinder is derived in this Appendix. Fig. 14 presents an outline of heat transfer across conductor together with the nomenclature used from thermal resistance calculation.

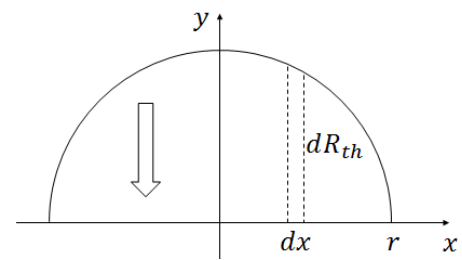

Fig. 14 Outline of heat transfer across conductor with nomenclature used for thermal resistance calculation.

An expression of the thermal resistance corresponding to the heat transferred across the small length $d x$, so called $d R$, can be given as follows:

$$
\begin{aligned}
& d R=\frac{y}{k(L \cdot d x)} \\
& \frac{1}{R}=\int_{-r}^{r} \frac{1}{d R}
\end{aligned}
$$

where, according to Pythagorean theorem,

$$
y=\sqrt{r^{2}-x^{2}}
$$

Thus,

$$
\begin{gathered}
\frac{1}{R}=k L \int_{-r}^{r} \frac{d x}{\sqrt{r^{2}-x^{2}}}=\left[k L \cdot \tan ^{-1}\left(\frac{x}{\sqrt{r^{2}-x^{2}}}\right)\right]_{-r}^{r} \\
\lim _{x \rightarrow+\infty} \tan ^{-1} x=\frac{\pi}{2} \\
\lim _{x \rightarrow-\infty} \tan ^{-1} x=-\frac{\pi}{2} \\
\frac{1}{R}=k l \pi
\end{gathered}
$$

Finally,

$$
R=\frac{1}{k L \pi}
$$

The thermal resistance across the conductor $R_{c}$ is obtained from (22),

$$
R_{c}=\frac{1}{\pi k_{c} L}
$$

Finally, following the lumped-parameter TEC representation shown in Fig. 5c), the equivalent winding thermal resistance $R_{e}$ is expressed:

$$
R_{e}=R_{i}+R_{c i}+R_{c}=\frac{\ln \frac{r_{i}}{r_{c i}}}{\pi k_{i} L}+\frac{\ln \frac{r_{c i}}{r_{c}}}{\pi k_{c i} L}+\frac{1}{\pi k_{c} L}
$$

The equivalent winding thermal conductivity is of interest here. According to (22),

$$
R_{e}=\frac{1}{\pi k_{e} L}
$$

Finally,

$$
k_{e}=\frac{1}{\frac{\ln \frac{r_{i}}{r_{c i}}}{k_{i}}+\frac{r_{c i}}{r_{c i}}+\frac{1}{k_{c i}}+\frac{1}{k_{c}}}
$$

\section{REFERENCES}

[1] R. Wrobel, P. Mellor, "A general cuboidal element for threedimensional thermal modelling," IEEE Trans. on Magnetics, vol. 46, no. 8, pp. 3197-3200, 2010.

[2] N. Simpson, R. Wrobel, P. H. Mellor, "Estimation of Equivalent Thermal Parameters of Impregnated Electrical Windings,"' IEEE Trans. on Industry Applications, vol. 49, no. 6, pp. 25052515, Nov. 2013.

[3] S. Ayat, R. Wrobel, J. Goss, D. Drury, "Estimation of Equivalent Thermal Conductivity for Impregnated Electrical Windings Formed from Profiled Rectangular Conductors", in 8th IET International Conference on Power Electronics, Machines and Drives (PEMD), Glasgow, April 2016.

[4] R. Wrobel, S. Ayat, J.L. Baker, "Analytical Methods for Estimating Equivalent Thermal Conductivity in Impregnated Electrical Windings Formed Using Litz Wire", International Electric Machines \& Drives Conference (IEMDC17), Miami, May 2017.

[5] S. Ayat, R. Wrobel, J. Goss, D. Drury, "Experiment Informed Methodology for Thermal Design of PM Machines", Eleventh International Conference on Ecological Vehicles and Renewable Energies (EVER), Monaco, April 2016.

[6] H. Liu, S. Ayat, R. Wrobel, C. Zhang, "Comparative Study of Thermal Properties of Electrical Windings Impregnated with Alternative Varnish Materials", in 9th IET International Conference on Power Electronics, Machines and Drives (PEMD), Liverpool, April 2018.

[7] R. Wrobel, N. Simpson, P. Mellor, J. Goss, D. Staton, "Design of a brushless PM starter-generator for low-cost manufacture and a high aspect-ratio mechanical space envelope", IEEE Energy Conversion And Exposition (ECCE), pp. 813-820, Sept. 2015.

[8] H. Liu, R. Wrobel, S. Ayat, C. Zhang, "Coupled Electromagnetic and Thermal Design Optimization of a Traction PM machine with High Torque Overload Capability", in International Conference on Electrical Machine (ICEM), 2016, in press.

[9] J. Widmer, C. Spargo, G. J. Atkinson, and B. C. Mecrow, "Solar plane propulsion motors with precompressed aluminum stator windings," IEEE Trans. Energy Conversion., vol. 29, no. 3, pp. 681-688, Sep. 2014.

[10] M. C. Kulan, N. J. Baker, and J. D. Widmer, "Design of a high fill factor permanent magnet integrated starter generator with compressed stator windings," IEEE Transactions on industry applications., vol. 53, no. 4, pp. 3371-3378, July/August 2017.

[11] J. Widmer, R. Martin, B.C .Mecrow, "Pre-Compressed and Stranded Aluminium Motor Windings for Traction Motors," IEEE International Machine and Drives Conference (IEMDC 2015), pp.1851,1857, May 2015.

[12] P. Romanazzi, M. Bruna, D. A. Howey, "Thermal homogenization of electrical machine windings applying the multiple-scales method," ASME Journal of Heat transfer, vol. 139, no. January, 2017.

[13] P. H. Mellor, D. Roberts, D. R. Turner. "Lumped parameter thermal model for electrical machines of TEFC design," Electric Power Applications, IEE Proceedings B, Vol. 138, No.5, pp.205-218, (1991).

[14] F. P. Incropera and D. P. Dewitt, Fundamentals of Heat and Mass Transfer (Wiley, New York, 1996).

[15] Z. Hashin and S. Shtrikman, "A variational approach to the theory of the effective magnetic permeability of multiphase materials," J. Appl. Phys., vol. 33, no. 10, pp. 3125-3131, Oct. 1962.

[16] A. Clifford, "Multivariate error analysis: a handbook of error propagation and calculation in many-parameter systems", John Wiley \& Sons, 1973.

[17] N. Simpson, R. Wrobel, P. H. Mellor, "A General Arc-Segment Element for Three-Dimensional Thermal Modeling," IEEE Transactions on Magnetics, vol. 50, no. 2, pp. 265-268, Feb. 2014

[18] J. L. Baker, R. Wrobel, D. Drury, and P. H. Mellor, "A methodology for predicting the thermal behaviour of modular-wound electrical machines," in IEEE Energy Conversion Congress and Exposition (ECCE), pp. 5176-5183, 2014.

[19] J. Pyrhonen, J. Tapani V. Hrabovcova, "Design of rotating electrical machines", John Wiley \& Sons, 2008.

[20] R. Wrobel, D. Salt, A. Griffo, N. Simpson, P. Mellor, "Derivation and scaling of AC copper loss in thermal modelling of electrical machines," IEEE Trans. Ind. Electron., vol. 61, no. 8, pp. 4412-4420, Aug. 2014

[21] https://www.safran-ventilation-systems.com/video/2098 (visited on $27 / 06 / 2018)$ 